Atıf için / For Citation: D. Osmaniye, B. Kaya Çavuşoğlu, "Potansiyel Antikolinesteraz Ajanlar Olarak Yeni Imidazol Türevlerinin Sentezi ve Karakterizasyonu”, Süleyman Demirel Üniversitesi Fen Edebiyat Fakültesi Fen Dergisi, 16(1), 301-307, 2021.

\title{
Potansiyel Antikolinesteraz Ajanlar Olarak Yeni İmidazol Türevlerinin Sentezi ve Karakterizasyonu
}

\author{
Derya OSMANIYE* ${ }^{* 1,2}$, Betül KAYA ÇAVUŞOĞLU²
}

${ }^{1}$ Anadolu Üniversitesi, Eczacllık Fakültesi, Farmasötik Kimya Anabilim Dall, 26470, Eskişehir, Türkiye

${ }^{2}$ Anadolu Üniversitesi, Eczacıllk Fakültesi, Doping ve Narkotik Maddeler Laboratuvarı, 26470, Eskişehir, Türkiye

${ }^{3}$ Bülent Ecevit Üniversitesi, Eczacllık Fakültesi, Farmasötik Kimya Anabilim Dall, 67600, Zonguldak, Türkiye

*dosmaniye@anadolu.edu.tr

(Alınış / Received: 04.11.2020, Kabul / Accepted: 21.05.2021, Yaylmlanma / Published: 27.05.2021)

Özet: Alzheimer hastalığ 1 en s1k görülen nörodejeneratif hastalıklarıdan biridir. Gelişmiş ülkelerde \%70-76 oranla görülen bu hastalığın radikal bir tedaviye sahip olmaması bilim dünyasını bu konu üzerinde çalışmaya yönlendirmektedir. Kolinerjik hipotez, AH hastalarında hafiza ve bilişsel bozukluğun ana nedeni düşük asetilkolin (ACh) düzeyinin olduğunu düşündürmektedir. Radikal bir tedavinin mevcut olmaması bilim insanları için bu alanı çekici kılmaktadır. İmidazol bileşikleri, çok çeşitli biyolojik aktiviteleri nedeniyle medisinal kimyada önemli bileşiklerdir. Bu çalışmada, yeni imidazol türevleri sentezlenmiş ve yapı tayinleri ${ }^{1} \mathrm{H}-$ NMR, ${ }^{13} \mathrm{C}-\mathrm{NMR}$ ve kütle spektroskopisi metodları kullanılarak aydınlatılmıştır. Bileşiklerin aktivite profilleri in vitro Ellman metodu kullanılarak gerçekleştirilmiştir. Aktivite sonucunda elde edilen veriler, $\mathbf{6}$ ve $\mathbf{7}$ kodlu bileşiklerin antikolinesteraz aktivite profillerinin umut vaat edici olduğunu ortaya koymuştur.

Anahtar kelimeler: İmidazol, Antikolinesteraz aktivite, NMR.

\section{Synthesis and Characterization of New Imidazole Derivatives as Potential Anticholinesterase Agents}

\begin{abstract}
Alzheimer's disease is one of the most common neurodegenerative diseases. This disease, which is seen with a rate of $70-76 \%$ in developed countries, does not have a radical treatment, which directs the scientific world to work on this issue. The cholinergic hypothesis suggests that low acetylcholine (ACh) levels are the main cause of memory and cognitive impairment in $\mathrm{AD}$ patients. The absence of a radical treatment makes this area attractive for scientists. Imidazole compounds are important compounds in medicinal chemistry due to their wide variety of biological activities. In this study, new imidazole derivatives were synthesized and the structure determinations were elucidated using ${ }^{1} \mathrm{H}-\mathrm{NMR},{ }^{13} \mathrm{C}-\mathrm{NMR}$ and mass spectroscopy methods. The activity profiles of the compounds were performed using the in vitro Elisa method. The data obtained as a result of the activity showed that the anticholinesterase activity profiles of the $\mathbf{6}$ and $\mathbf{7}$ coded compounds were promising.
\end{abstract}

Key words: Imidazole, Anticholinesterase activity, NMR. 


\section{Giriş}

Alzheimer hastalığı (AH) şu anda dünyadaki nörodejeneratif demansın en yaygın nedenidir. Demans vakalarının yaklaşık \%70-76'sına gelişmiş ülkelerde rastlanmaktadır. $\mathrm{Bu}$ oran gelişmiş ülkelerde uzun ömürlü yaşamaya bağlanmaktadır. Bunlar haricinde $\mathrm{AH}, \beta$-amiloid içeren plaklar, tau içeren nörofibriler düğümler ve kolinerjik nöronların kaybı ile patolojik olarak karakterize edilen yaşa bağlı bir hastalıktır. Hastalık belirtileri arasında hafiza kaybı, psikoz ve depresyon bulunur. Her ne kadar kolinerjik nöronal kayıp hastalığın karakteristik özelliği olsa da, kolinerjik sistemlerle ilgili tedaviler, diğer yolların da tutulumunu gösteren hastalığın ilerlemesini önleyemez [1-5].

AH patogenezinin mekanizmasını açıklamak için kolinerjik hipotez, amiloid hipotezi gibi çeşitli hipotezler önerilmiştir. Kolinerjik hipotez, AH hastalarında hafıza ve bilişsel bozukluğunun ana nedeni düşük asetilkolin ( $\mathrm{ACh}$ ) düzeyinin olduğunu düşündürmektedir. Çalışmaların çoğu, asetilkolinesterazın (AChE) kolinerjik sinapslarda nörotransmitter ACh'yi hidrolize ederek çeşitli fizyolojik reaksiyonların düzenlenmesinde önemli bir rol oynadığını göstermektedir. Hastalık semptomlarını iyileştirmek için en etkili strateji, asetilkolinesteraz (AChE) ve butirilkolinesteraz (BChE) inhibitörleri olarak adlandırılan kolinesterazlar (ChEI'ler) kullanarak asetilkolin (ACh) seviyesini yükseltmektir. Şimdiye kadar, FDA tarafından onaylanan mevcut beş AH ilacının dördü, rivastigmin, galantamin ve donepezilin klinik kullanımda olan ChE inhibitörleridir. Bu nedenle, yeni ChEI'lerinin keşfi, AH semptomatik tedavisi için hala en değerli ve baskın hedeflerden birini temsil etmektedir [6,7].

İmidazol, birçok doğal bileşiğin yapısında bulunması nedeniyle ve önemli fizyolojik olaylarda rol alan maddelerin (histidin, histamin, B12 vitamini) yapısında bulunmasından dolayı önemli bir heterosiklik halkadır ve insan organizmasının yabancı olmadığı bir maddedir [8].

İmidazol ve türevleri tıpta ve veterinerlikte yaygın olarak kullanılan bileşiklerdir. İmidazol ve türevlerinin yapılan çalışmalar sonucu canlı metabolizmasındaki farklı görevleri ortaya konulmuştur. İmidazol türevlerinin literatür kayıtları arasında antikanser [9,10], antifungal [11] etkileri yanında antikolinesteraz etkileri ilgili çalışmaların varlığı $[12,13]$ ve günümüzde kullanılan birçok ilaç molekülünün yapısına girmiş olması bizi bu tür çalışmaya yönlendirmiştir. [8,14]. Bu bilgiler 1şı̆̆ında 6 adet yeni imidazol bileşiği sentezlenmiş ve in vitro yöntemler kullanılarak antikolinesteraz aktiviteleri değerlendirilmiştir.

\section{Materyal ve Metot}

Çalışmadaki bileşiklerin sentezi Anadolu Üniversitesi Eczacılık Fakültesi Farmasötik Kimya Araştırma Laboratuvarında, Bileşiklerin analizi ve aktivitesi ise Anadolu Üniversitesi Eczacılık Fakültesi Doping ve Narkotik Maddeler Analiz Laboratuvarında gerçekleştirilmiştir.

\subsection{Sentez Çalışmalart}

\subsubsection{4-(4-(2-hidroksietil)piperazin-1-il)benzaldehid sentezi (1)}

2-(Piperazin-1-il)etan-1-ol (1,04 g, 0,008 mol) $30 \mathrm{~mL}$ hacimli balon içerisine alınmış üzerine $10 \mathrm{~mL} \mathrm{DMF}, \mathrm{K}_{2} \mathrm{CO}_{3}(1,10 \mathrm{~g}, 0,008 \mathrm{~mol})$ ve 4-florobenzaldehid $(0,99 \mathrm{~g}, 0,008$ mol) ilave edilmiştir. Reaksiyon karışımı, geri çeviren soğutucu altında $170{ }^{\circ} \mathrm{C}$ sicaklıkta 36 saat tutulmuştur. Reaksiyon süresi bitiminde viyal içeriği buzlu suya 
dökülerek ürün çöktürülmüş, bol su ile yıkanmış, kurutulmuş ve etanolden kristallendirilmiştir.

\subsubsection{Hedef bileşiklerin sentezi (2-7)}

1,2-di-p-sübstitüteetan-1,2-dion $(0,002 \mathrm{~mol})$ asetik asit içerisinde çözülmüştür. Hazırlanan çözeltinin içerisine aldehid türevleri $(0,002 \mathrm{~mol})$ ve amonyum asetat $(0,0024$ mol) ilave edilerek mikrodalga sentez reaktörüne alınmış ve $200{ }^{\circ} \mathrm{C}$ sıcaklık ve 10 bar basınç altında 30 dakika tutulmuştur. Reaksiyon süresi bitiminde vial içeriği buzlu suya dökülerek ürün çöktürülmüş, bol su ile yıkanmış, kurutulmuş ve etanolden kristallendirilmiştir.

\section{1-Metil-4',5'-difenil-1H,1'H-2,2'-biimidazol (2)}

Yield: $75 \%,{ }^{1} \mathrm{H}-\mathrm{NMR}\left(300 \mathrm{MHz}, \mathrm{DMSO}-d_{6}\right): \delta=4.12\left(3 \mathrm{H}, \mathrm{s},-\mathrm{CH}_{3}\right), 7.03(1 \mathrm{H}, \mathrm{d}$, $J=1.1 \mathrm{~Hz}$, İmidazol), $7.31(1 \mathrm{H}, \mathrm{d}, J=1.0 \mathrm{~Hz}$, İmidazol $), 7.32-7.35(5 \mathrm{H}, \mathrm{m}$, Monosübstitüebenzen), 7.50-7.53 (5H, m, Monosübstitüebenzen), 13.07 (1H, s, -NH). ${ }^{13} \mathrm{C}-\mathrm{NMR}\left(75 \mathrm{MHz}, \mathrm{DMSO}-d_{6}\right): \delta=31.3,121.9,127.5,128.1,128.7,129.1,129.3$, 138.2, 157.9. ESI-MS $[\mathrm{M}+\mathrm{H}]+: 301$

\section{1-Metil-4',5'-di-p-tolil-1H,1'H-2,2'-biimidazol (3)}

Yield: $79 \%, 1 \mathrm{H}-\mathrm{NMR}\left(300 \mathrm{MHz}, \mathrm{DMSO}-d_{6}\right): \delta=2.31\left(6 \mathrm{H}, \mathrm{s},-\mathrm{CH}_{3}\right), 4.10(3 \mathrm{H}, \mathrm{s},-$ $\mathrm{CH}_{3}$ ), 7.02 (1H, d, $J=1.0 \mathrm{~Hz}$, İmidazol), 7.12-7.16 (4H, m, 1,4-Disübstitüebenzen), 7.29 $(1 \mathrm{H}, \mathrm{d}, J=1.0 \mathrm{~Hz}$, İmidazol), 7.36-7.39 (4H, m, 1,4-Disübstitüebenzen), $12.93(1 \mathrm{H}, \mathrm{s},-$ $\mathrm{NH}) .{ }^{13} \mathrm{C}-\mathrm{NMR}\left(75 \mathrm{MHz}, \mathrm{DMSO}-d_{6}\right): \delta=21.3,35.2,124.1,127.6,127.9,128.7,128.8$, $129.3,129.3,129.5,129.5,136.1,137.4,138.5,139.3$. ESI-MS $[\mathrm{M}+\mathrm{H}]+: 329$

\section{2-(Benzo[d][1,3]dioksol-5-il)-4,5-difenil-1H-imidazol (4)}

Yield: $81 \%, 1 \mathrm{H}-\mathrm{NMR}\left(300 \mathrm{MHz}, \mathrm{DMSO}-d_{6}\right): \delta=6.09$ (2H, s, Benzo[d][1,3]dioksol), $7.03(1 \mathrm{H}, \quad \mathrm{d}, \quad J=8.6 \mathrm{~Hz}, \quad$ Benzo[d][1,3]dioksol), 7.21-7.24 $(1 \mathrm{H}, \quad \mathrm{m}$, Monosübstitüebenzen), $7.30(2 \mathrm{H}, \mathrm{t}, J=7.6 \mathrm{~Hz}$, Monosübstitüebenzen), 7.36-7.38 (1H, m, Monosübstitüebenzen), $7.44(2 \mathrm{H}, \mathrm{t}, J=7.5 \mathrm{~Hz}$, Monosübstitüebenzen), 7.49 (2H, d, $J=7.1 \mathrm{~Hz}$, Monosübstitüebenzen), $7.54(2 \mathrm{H}, \mathrm{d}, J=7.2 \mathrm{~Hz}$, Monosübstitüebenzen), 7.61 $(1 \mathrm{H}, \mathrm{s}$, Benzo[d][1,3]dioksol), 7.62-7.64 $(1 \mathrm{H}, \mathrm{m}$, Benzo[d][1,3]dioksol), $12.51(1 \mathrm{H}, \mathrm{s},-$ $\mathrm{NH}) .{ }^{13} \mathrm{C}-\mathrm{NMR}\left(75 \mathrm{MHz}, \mathrm{DMSO}-d_{6}\right): \delta=101.74,105.99,109.03,119.75,125.11$, $126.93,127.52,128.15,128.26,128.63,128.82,129.11,129.63,131.59,135.67$, 137.23, 145.86, 147.87, 148.10. ESI-MS [M+H]+ : 341

\section{2-(Benzo[d][1,3]dioksol-5-il)-4,5-di-p-tolil-1H-imidazol (5)}

Yield: $77 \%,{ }^{1} \mathrm{H}-\mathrm{NMR}\left(300 \mathrm{MHz}, \mathrm{DMSO}-d_{6}\right): \delta=2.29\left(3 \mathrm{H}, \mathrm{s},-\mathrm{CH}_{3}\right), 2.34(3 \mathrm{H}, \mathrm{s},-$ $\left.\mathrm{CH}_{3}\right), \quad 6.08 \quad(2 \mathrm{H}, \quad \mathrm{s}, \quad$ Benzo[d][1,3]dioksol), $7.01 \quad(1 \mathrm{H}, \quad \mathrm{d}, \quad J=8.6 \mathrm{~Hz}$, Benzo[d][1,3]dioksol), 7.09-7.11 (2H, m, 1,4-Disübstitüebenzen), 7.21-7.24 (2H, m, 1,4-Disübstitüebenzen), 7.36-7.40 (4H, m, 1,4-Disübstitüebenzen), $7.59(1 \mathrm{H}, \mathrm{s}$, Benzo[d][1,3]dioksol), 7.60-7.62 (1H, m, Benzo[d][1,3]dioksol), $12.38(1 \mathrm{H}, \mathrm{s},-\mathrm{NH})$. ${ }^{13} \mathrm{C}-\mathrm{NMR}\left(75 \mathrm{MHz}, \mathrm{DMSO}-d_{6}\right): \delta=21.28,101.70,105.93,109.00,119.65,121.74$, $125.21,127.44,128.59,128.76,129.20,129.62,132.92,135.93,137.02,137.35$, 145.52, 147.76, 148.07. ESI-MS [M+H]+ : 369.

\section{2-(4-(4-(4,5-diphenyl-1H-imidazol-2-yl)phenyl)piperazin-1-yl)ethan-1-ol (6)}

Yield: $81 \%,{ }^{1} \mathrm{H}-\mathrm{NMR}\left(300 \mathrm{MHz}, \mathrm{DMSO}-d_{6}\right): \delta=2.45\left(2 \mathrm{H}, \mathrm{t}, J=6.2 \mathrm{~Hz},-\mathrm{CH}_{2}-\right), 2.56$ $(4 \mathrm{H}, \mathrm{t}, J=4.6 \mathrm{~Hz}$, piperazin), $3.21(4 \mathrm{H}, \mathrm{t}, J=4.4 \mathrm{~Hz}$, piperazin), $3.55(2 \mathrm{H}, \mathrm{q}, J=4.6 \mathrm{~Hz},-$ CH2-), $4.48(1 \mathrm{H}, \mathrm{y},-\mathrm{OH}), 7.01(2 \mathrm{H}, \mathrm{d}, J=8.9 \mathrm{~Hz}, 1,4$-Disübstitüebenzen), 7.20-7.23 (1H, m, Monosübstitüebenzen), $7.29(2 \mathrm{H}, \mathrm{t}, J=7.3 \mathrm{~Hz}$, Monosübstitüebenzen), 7.34$7.36(1 \mathrm{H}, \mathrm{m}$, Monosübstitüebenzen), $7.42(2 \mathrm{H}, \mathrm{t}, J=7.1 \mathrm{~Hz}$, Monosübstitüebenzen), 7.49 
$(2 \mathrm{H}, \quad \mathrm{d}, \quad J=7.4 \mathrm{~Hz}, \quad$ Monosübstitüebenzen), $7.55 \quad(2 \mathrm{H}, \quad \mathrm{d}, \quad J=7.3 \mathrm{~Hz}$, Monosübstitüebenzen), $7.94(2 \mathrm{H}, \mathrm{d}, J=8.9 \mathrm{~Hz}, 1,4$-Disübstitüebenzen), $12.41(1 \mathrm{H}, \mathrm{s},-$ $\mathrm{NH}) .{ }^{13} \mathrm{C}-\mathrm{NMR}\left(75 \mathrm{MHz}, \mathrm{DMSO}-d_{6}\right): \delta=48.08,53.58,59.05,60.76,115.16,121.17$, $126.66,126.80,127.53,127.77,127.95,128.60,128.76,129.06,131.79,135.91$, 137.11, 146.57, 151.28. ESI-MS [M+H]+ : 425.

\section{2-(4-(4-(4,5-di-p-tolyl-1H-imidazol-2-yl)phenyl)piperazin-1-yl)ethan-1-ol (7)}

Yield: $81 \%,{ }^{1} \mathrm{H}-\mathrm{NMR}\left(300 \mathrm{MHz}, \mathrm{DMSO}-d_{6}\right): \delta=2.29\left(3 \mathrm{H}, \mathrm{s},-\mathrm{CH}_{3}\right), 2.34(3 \mathrm{H}, \mathrm{s},-$ $\left.\mathrm{CH}_{3}\right), 2.44(2 \mathrm{H}, \mathrm{t}, J=6.2 \mathrm{~Hz},-\mathrm{CH} 2-), 2.56(4 \mathrm{H}, \mathrm{t}, J=4.7 \mathrm{~Hz}$, piperazin), $3.21(4 \mathrm{H}, \mathrm{t}$, $J=4.4 \mathrm{~Hz}$, piperazin), $3.55\left(2 \mathrm{H}, \mathrm{t}, J=6.2 \mathrm{~Hz},-\mathrm{CH}_{2}-\right), 4.47(1 \mathrm{H}, \mathrm{y},-\mathrm{OH}), 7.00(2 \mathrm{H}, \mathrm{d}$, $J=9.0 \mathrm{~Hz}, 1,4$-Disübstitüebenzen), 7.09 (2H, d, $J=8.2 \mathrm{~Hz}, 1$,4-Disübstitüebenzen), 7.21 $(2 \mathrm{H}, \mathrm{d}, \quad J=7.5 \mathrm{~Hz}, \quad$ 1,4-Disübstitüebenzen $), 7.36 \quad(2 \mathrm{H}, \mathrm{d}, \quad J=7.6 \mathrm{~Hz}, \quad 1,4-$ Disübstitüebenzen), 7.42 ( $2 \mathrm{H}, \mathrm{d}, J=7.5 \mathrm{~Hz}, 1,4$-Disübstitüebenzen), 7.90 (2H, d, $J=8.9$

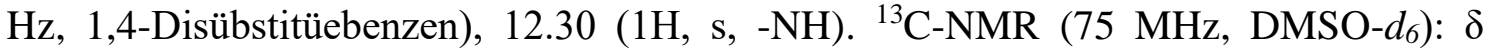
$=21.26,48.11,53.58,59.05,60.76,115.15,121.33,126.58,127.44,128.55,128.95$, $129.16,129.58,133.18,135.75,136.84,137.10,146.22,151.19$. ESI-MS $[\mathrm{M}+\mathrm{H}]+$ : 453.

\subsection{Antikolinesteraz Aktivite}

Tüm bileşikler, ChE enzimlerini inhibe etme güçlerini değerlendirmek için daha önce ekibimiz tarafından rapor edilmiş modifiye Elmann testi yöntemine tabi tutulmuştur [15-17].

\section{AChE enzim solüsyonunun hazırlanması}

Liyofilize haldeki AChE enzimini çözmek için \%1'lik jelatin çözeltisi hazırlanmıştır. AChE enzimi jelatin çözeltisinde $500 \mathrm{U} / \mathrm{mL}$ konsantrasyonda hazırlanmıştır. Enzim çözeltisinden $1 \mathrm{~mL}$ alınarak balon joje içerisinde hacim suyla $100 \mathrm{~mL}$ 'ye tamamlanmıştır. Böylelikle $5 \mathrm{U} / \mathrm{mL}$ 'ye seyreltilmiş stok enzim solüsyonu hazırlanmıştır. Hazırlanan stok çözelti $0,7 \mathrm{~mL}$ 'lik porsiyonlar halinde $-20{ }^{\circ} \mathrm{C}$ 'de saklanmıştır. Enzim çözeltileri, aktivite çalışmalarına başlamadan önce oda isısına getirilmiş ve metod konsantrasyonu olan 2,5 U/mL'ye seyreltmek için suyla 1,4 mL'ye tamamlanarak kullanılmıştır.

Asetiltiyokolin iyodür (ATC) çözeltisinin hazırlanması (0,075 M)

ATC $(0,217 \mathrm{~g})$ bir miktar suda çözülmüş ardından suyla $10 \mathrm{~mL}$ 'ye tamamlanmıştır. Hazırlanan çözelti, kullanılıncaya kadar $0,4 \mathrm{~mL}$ 'lik kısımlar halinde $-20{ }^{\circ} \mathrm{C}$ 'de saklanmıştır.

\section{5-5-ditiyobis(2-nitrobenzoik asit) (DTNB) solüsyonunun hazırlanması (0,01 M)}

DTNB $(0,396 \mathrm{~g})$ tartılarak bir miktar suda çözülmüştür. Bu çözeltiye sodyum bikarbonat $(0,15 \mathrm{~g})$ ilave edilmiş ve hacim suyla $100 \mathrm{~mL}$ 'ye tamamlanmıştır. Hazırlanan çözelti, kullanılıncaya kadar $3 \mathrm{~mL}$ 'lik kısımlar halinde $-20{ }^{\circ} \mathrm{C}$ 'de saklanmıştır.

Fosfat tamponunun hazırlanmasi $(p H=8,0)$

Potasyum dihidrojen fosfat $(13,61 \mathrm{~g}), 1$ L suda çözülmüştür. Hazırlanan çözeltinin pH'sı 0,1 N potasyum hidroksit çözeltisi ile pH metre kullanılarak kontrollü biçimde $8,0+0,1$ 'e ayarlanmıştır. Ayarlı tampon çözelti $0,22 \mu \mathrm{m}$ por çapı olan tek kullanımlık filtrelerden süzülerek kullanıma hazır hale getirilmiştir. Hazırlanan çözelti, kullanılıncaya kadar $4{ }^{\circ} \mathrm{C}$ 'de saklanmıştır. Enzim solüsyonu, inhibitör madde solüsyonu, fosfat tamponu ve 5,5-ditiyobis(2-nitrobenzoik asit) çok kanallı mikropipetler yardımıyla 96 kuyucuklu plakalara konmuştur. Karışım $25{ }^{\circ} \mathrm{C}$ 'de 15 dakika inkübasyona bırakılmıştır. Bu süre sonunda enzim substrat reaksiyonu, ATC 
ilavesi ile başlatılarak, mikroplaka okuyucu ile 412 nm'de beş dakika aralıkla iki absorbsiyon ölçümü gerçekleştirilmiştir. Referans olarak inhibitör madde olmaksızın aynı işlem gerçekleştirilerek okuma yapılmıştır. Kontrol ajanı olarak AChE inhibitörü donezepil kullanılmıştır. Enzim inhibisyonları aşağıdaki eşitlik kullanılarak hesaplanmıştır.

$$
\% \mathrm{EI}=[(\text { Akonts }- \text { Akonti })-(\text { Ainhs }- \text { Ainhi }) /(\text { Akonts }- \text { Akonti })] \times 100
$$

$\%$ Eİ = Yüzde enzim inhibisyonu Ainhs = İnhibitör varlığındaki beşinci dakika absorbansı Ainhi = İnhibitör varlığındaki sıfırıncı dakika absorbansı

Akonts $=$ Referans solüsyonun (inhibitörsüz) beşinci dakika absorbans1

Akonti $=$ Referans solüsyonun (inhibitörsüz) sıfırıncı dakika absorbansı

Bütün absorbans değerleri boş okunan (blank) değerleri ile doğrulanmıştır. Her bir deney dört bağımsız kuyucukta gerçekleştirilmiş ve ölçümlerinde ortalama absorbans değerleri kullanılarak \% enzim inhibisyon değerleri hesaplanmıştır.

\section{Bulgular}

\subsection{Sentez Çalışmaları}

$\mathrm{Bu}$ çalışma kapsamında 6 adet imidazol türevi sentezlenmiş ve antikolinesteraz aktiviteleri incelenmiştir. Hedef bileşiklere ait sentez şeması Şekil-1'de sunulmuştur. İlk olarak 6 ve 7 nolu türevlerin sentezinde kullanılmak üzere 4-(4-(2hidroksietil)piperazin-1-il)benzaldehid (1) bileşiği elde edilmiştir. Daha sonra elde edilen aldehid türevi (1), 1-metil-1H-imidazol-2-karbaldehid ve benzo[d][1,3]dioksol-5karbaldehid ile 1,2-di-p-sübstitüteetan-1,2-dion türevleri mikrodalga sentez reaktörü yardımıyla reaksiyona sokularak hedef bileşiklerin (2-7) sentezi tamamlanmıştır. Bu çalışma kapsamında sentezi gerçekleştirilen 6 adet yeni imidazol türevinin yapıları ${ }^{1} \mathrm{HNMR},{ }^{13} \mathrm{C}-\mathrm{NMR}$ ve mass spektroskopik verileri yardımıyla aydınlatılmıştır.

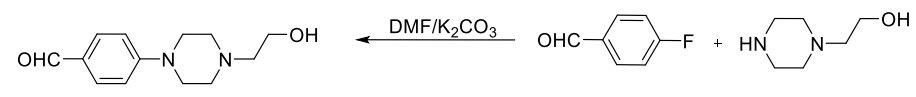

(1)
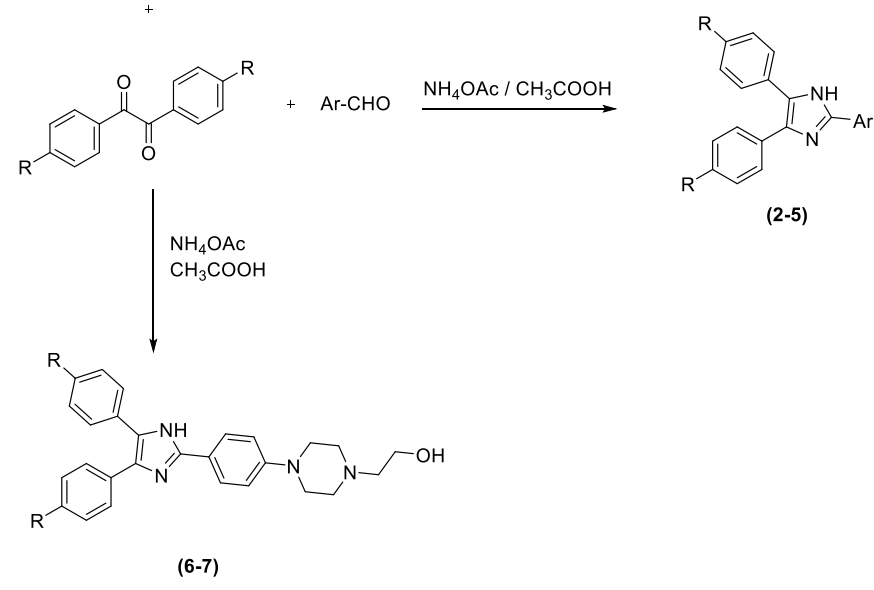

\begin{tabular}{ccc}
\hline Bileşik & $\mathbf{R}$ & $\mathbf{A r}$ \\
\hline $\mathbf{2}$ & $-\mathrm{H}$ & 1-Metilimidazol-2il \\
$\mathbf{3}$ & $-\mathrm{CH}_{3}$ & 1-Metilimidazol-2il \\
$\mathbf{4}$ & $-\mathrm{CH}_{3}$ & Benzo[d][1,3]dioksol-5-il
\end{tabular}




\begin{tabular}{ccc}
$\mathbf{5}$ & $-\mathrm{H}$ & Benzo[d][1,3]dioksol-5-il \\
$\mathbf{6}$ & $-\mathrm{H}$ & - \\
$\mathbf{7}$ & $-\mathrm{CH}_{3}$ & - \\
\hline
\end{tabular}

Şekil 1: Hedef bileşiklerin (2-7) sentez şeması

\subsection{Antikolinesteraz Aktivite}

Daha önce grubumuz tarafindan çalışılan modifiye Ellman metoduna göre in vitro AChE ve BChE aktiviteleri incelenmiştir [15-17].

Elde edilen verilere göre her iki konsantrasyon $\left(10^{-3} \mathrm{M}\right.$ ve $\left.10^{-4} \mathrm{M}\right)$ değerinde $\% 50$ üzerinde aktivite gösterebilen bileşik gözlenmemiştir. Fakat 4-(4-(2hidroksietil)piperazin-1-il)benzaldehid kullanılarak elde edilen türevler umut verici aktivite sonuçları sergilemiştir. 6 numaralı bileşik sırasıyla $10^{-3} \mathrm{M}$ ve $10^{-4} \mathrm{M}$ konsantrasyonda \% 56.20 ve \% 42.98 inhibisyon değerleri ile aktivite göstermiştir. 7 numaralı bileşik ise sirasıyla $10^{-3} \mathrm{M}$ ve $10^{-4} \mathrm{M}$ konsantrasyonda $\% 51.89$ ve $\% 45.15$ inhibisyon değerleri ile aktivite göstermiştir. Ayrıca bu bileşiklerin asetilkolinesteraz enzimine karşı seçicilik göstermeleri dikkat çekmektedir. Bu bilgiler 1şığında piperazin halkasının yapıya katılmasının aktiviteyi pozitif önde etkilediği görülmektedir. İleri çalışmalarda piperazin halkasının sübstitüsyonu değiştirilerek antikolinesteraz aktivite potansiyeli daha yüksek türevler sentezlenmesi planlanmaktadır.

Tablo 1. 2-7 Kodlu Bileşiklere Ait \%AChE ve \%BChE İnhibisyon Değerleri

\begin{tabular}{|l|c|c|c|c|}
\hline \multirow{2}{*}{ Bileşik } & \multicolumn{2}{|c|}{$\mathbf{A C h E ~ \% ~ I ̇ n h i b i s y o n u ~}$} & \multicolumn{2}{c|}{ BChE \% Inhibisyonu } \\
\cline { 2 - 5 } & $\mathbf{1 0}^{-\mathbf{3}} \mathbf{M}$ & $\mathbf{1 0}^{-\mathbf{4}} \mathbf{M}$ & $\mathbf{1 0}^{-\mathbf{3}} \mathbf{M}$ & $\mathbf{1 0}^{-\mathbf{4}} \mathbf{M}$ \\
\hline $\mathbf{2}$ & $25.20 \pm 0.22$ & $19.58 \pm 0.21$ & $21.53 \pm 0.38$ & $19.32 \pm 0.28$ \\
\hline $\mathbf{3}$ & $35.28 \pm 0.87$ & $31.90 \pm 0.58$ & $27.40 \pm 0.59$ & $19.34 \pm 0.29$ \\
\hline $\mathbf{4}$ & $42.45 \pm 0.77$ & $39.20 \pm 0.51$ & $31.96 \pm 0.25$ & $25.65 \pm 0.45$ \\
\hline $\mathbf{5}$ & $39.70 \pm 0.25$ & $28.55 \pm 0.76$ & $45.96 \pm 0.38$ & $33.15 \pm 0.36$ \\
\hline $\mathbf{6}$ & $\mathbf{5 6 . 2 0} \pm \mathbf{0 . 6 0}$ & $\mathbf{4 2 . 9 8} \pm \mathbf{0 . 5 5}$ & $31.79 \pm 0.61$ & $24.86 \pm 0.38$ \\
\hline $\mathbf{7}$ & $\mathbf{5 1 . 8 9} \pm \mathbf{0 . 9 5}$ & $\mathbf{4 5 . 1 5} \pm \mathbf{0 . 6 6}$ & $40.29 \pm 0.58$ & $31.59 \pm 0.25$ \\
\hline
\end{tabular}

\section{Sonuç ve Yorum}

Özetle bu çalışma kapsamında mikrodalga sentez reaktörü kullanılarak yeni imidazol türevleri sentezlenmiş, bileşiklerin yap1 tayinler ${ }^{1} \mathrm{H}-\mathrm{NMR},{ }^{13} \mathrm{C}-\mathrm{NMR}$ ve Mass spektroskopik analizleri yapılarak tamamlanmış ve bu bileşiklerin in vitro antikolinesteraz aktiviteleri incelenmiştir. Bileşiklerin hiçbiri bütirilkolinesteraz inhibisyonu göstermezken $\mathbf{6}$ ve $\mathbf{7}$ kodlu bileşikler asetilkolinesteraz enzimine karşı umut vaat edici düzeyde aktivite göstermişlerdir. $\mathrm{Bu}$ çalışmadan elde edilen veriler antikolinesteraz aktivite potansiyeli yüksek türevler geliştirmek adına umut vericidir.

\section{Araştırmacıların Katkı Oranı Beyanı}

Derya Osmaniye: Metodoloji, Araştırma, Kaynak/Materyal/Malzeme Temini, Orijinal Taslak Yazımı Betül KAYA ÇAVUŞOĞLU: Metodoloji, Biçimsel Analiz, İnceleme ve Düzenleme

\section{Destek ve Teşekkür Beyanı}

Bu çalışmanın yazarları olarak Anadolu Üniversitesi Eczacılık Fakültesi Doping ve Narkotik Maddeler Laboratuvarına destek ve katkılarından dolayı teşekkür ederiz.

\section{Çatışma Beyanı}

$\mathrm{Bu}$ çalışmanın yazarları olarak herhangi bir çatışma beyanımız bulunmadığını bildiririz.

\section{Etik Kurul Onayı ve/veya Aydınlatılmış Onam Bilgileri}


$\mathrm{Bu}$ çalışmanın yazarları olarak herhangi bir etik kurul onayı ve/veya aydınlatılmış onam bilgileri beyanımız bulunmadığını bildiririz.

\section{Kaynakça}

[1] A. Mitelpunkt, T. Galili, T. Kozlovski, N. Bregman, N. Shachar, M. Markus-Kalish, Y. Benjamini, "Novel Alzheimer's disease subtypes identified using a data and knowledge driven strategy," Sci. Rep., 10(1), 1-13, 2020.

[2] M.L.B. Pulido, J.B.A. Hernández, M.A.F. Ballester, C.M.T. González, J. Mekyska, Z. Smékal "Alzheimer's disease and automatic speech analysis: a review," Expert Syst. Appl., 113213, 2020.

[3] S. Cordone, L. De Gennaro, "Insights from human sleep research on neural mechanisms of Alzheimer's disease," Neural Regen. Res., 15(7), 1251, 2020.

[4] S. Singh, "Noradrenergic pathways of locus coeruleus in Parkinson's and Alzheimer's pathology," Int. J. Neurosci., 130(3), 251-261, 2020.

[5] C. Bellenguez, B. Grenier-Boley, J.C. Lambert, "Genetics of Alzheimer's disease: where we are, and where we are going," Curr. Opin. Neurol., 61, 40-48, 2020.

[6] Y. Xu, Z. Zhang, X. Jiang, X. Chen, Z. Wang, H. Alsulami, W. "Tang. Discovery of $\delta$-sultone-fused pyrazoles for treating Alzheimer's disease: Design, synthesis, biological evaluation and SAR studies,” Eur. J. Med. Chem., 181, 111598, 2019.

[7] P. Bai, K. Wang, P. Zhang, J. Shi, X. Cheng, Q. Zhang, Z. Sang, "Development of chalcone-Oalkylamine derivatives as multifunctional agents against Alzheimer's disease,"Eur. J. Med. Chem., 183, 111737, 2019.

[8] Ö. Keskin, "İmidazolidin-2-tiyon türevlerinin sentezi, karakterizasyonu, dft ve doking hesaplamaları," Yüksek Lisans Tezi, Türkiye, Adıyaman Üniversitesi, Adıyaman, 2018.

[9] Wang, R. Shi, H. F. Zhao, J. F. He, Y. P. Zhang, H. B. Liu, J. P. 'Design, synthesis and aromatase inhibitory activities of novel indole-imidazole derivatives.' Bioorg. Med. Chem. Lett., 23(6), 17601762, 2013.

[10] Di Matteo, M. Ammazzalorso, A. Andreoli, F. Caffa, I. De Filippis, B. Fantacuzzi, M., Soncini, D. "'Synthesis and biological characterization of 3-(imidazol-1-ylmethyl) piperidine sulfonamides as aromatase inhibitors,'” Bioorg. Med. Chem. Lett., 26(13), 3192-3194, 2016.

[11] Al-Wabli, R. I. Al-Ghamdi, A. R. Aswathy, S. A. V. Ghabbour, H. A. Al-Agamy, M. H. Hubert Joe, I. Attia, M. I. ''(2E)-2-[1-(1, 3-Benzodioxol-5-yl)-3-(1H-imidazol-1-yl) propylidene]-N-(2chlorophenyl) hydrazine carboxamide: Synthesis, X-ray Structure, Hirshfeld Surface Analysis, DFT Calculations, Molecular Docking and Antifungal Profile,' Crystals, 9(2), 82, 2019.

[12] Vitorović-Todorović, M. D. Koukoulitsa, C. Juranić, I. O. Mandić, L. M., Drakulić, B. J. 'SStructural modifications of 4-aryl-4-oxo-2-aminylbutanamides and their acetyl-and butyrylcholinesterase inhibitory activity. Investigation of AChE-ligand interactions by docking calculations and molecular dynamics simulations,' Eur. J. Med. Chem., 81, 158-175, 2014.

[13] Xu, Y. X. Wang, H. Li, X. K. Dong, S. N. Liu, W. W. Gong, Q. .. Mao, F. '’Discovery of novel propargylamine-modified 4-aminoalkyl imidazole substituted pyrimidinylthiourea derivatives as multifunctional agents for the treatment of Alzheimer's disease'” Eur. J. Med. Chem., 143, 33-47, 2018.

[14] Lombardino JG. U.S. Patent No. 3,707,475. Washington, DC: U.S. Patent and Trademark Office 1972.

[15] G.L. Ellman, K.D. Courtney, Jr. V. Andres, R.M. Featherstone, "A new and rapid colorimetric determination of acetylcholinesterase activity," Biochem. Pharmacol., 7, 88-95, 1961.

[16] U. Acar Çevik, B.N. Saglık, S. Levent, D. Osmaniye, B. Kaya Çavusoglu, Y. Özkay, Z.A. Kaplancikl1, "Synthesis and AChE-inhibitory activity of new benzimidazole derivatives," Molecules, 24, 861, 2019.

[17] D. Osmaniye, B.N. Sağlık, U. Acar Çevik, S. Levent, B. Kaya Çavuşoğlu, Y. Özkay, G. Turan, "Synthesis and AChE inhibitory activity of novel thiazolylhydrazone derivatives," Molecules, 24(13), 2392, 2019. 